\title{
An Open-Label Investigation of the Pharmacokinetics and Tolerability of Oral Cysteamine in Adults with Cystic Fibrosis
}

\author{
Graham Devereux $^{1,5} \cdot$ Sandra Steele $^{1} \cdot$ Kairen Griffiths $^{1} \cdot$ Edward Devlin $^{1,2}$ • \\ Douglas Fraser-Pitt ${ }^{2} \cdot$ Seonaidh Cotton $^{3} \cdot$ John Norrie $^{3} \cdot$ Henry Chrystyn $^{4}$. \\ Deborah O'Neil ${ }^{2}$
}

Published online: 6 May 2016

(C) The Author(s) 2016. This article is published with open access at Springerlink.com

\begin{abstract}
Background and Objective Cysteamine is licensed for use in nephropathic cystinosis but preclinical data suggest a role in managing cystic fibrosis $(\mathrm{CF})$. This study aimed to determine whether oral cysteamine is absorbed in adult $\mathrm{CF}$ patients and enters the bronchial secretions. Tolerability outcomes were also explored.

Methods Patients $\geq 18$ years of age, weighing $>50 \mathrm{~kg}$ with stable CF lung disease were commenced on oral cysteamine bitartrate $\left(\right.$ Cystagon $\left.^{\circledR}\right) 450 \mathrm{mg}$ once daily, increased weekly to $450 \mathrm{mg}$ four times daily. Serial plasma cysteamine concentrations were measured for $24 \mathrm{~h}$ after the first dose. Participants were reviewed every week for 6 weeks, except at 4 weeks. Plasma cysteamine
\end{abstract}

Graham Devereux

g.devereux@abdn.ac.uk

1 Cystic Fibrosis Clinic, Aberdeen Royal Infirmary, Foresterhill, Aberdeen, UK

2 Novabiotics Ltd, Cruickshank Building, Craibstone, Aberdeen, UK

3 Centre for Healthcare Randomised Trials, University of Aberdeen, Health Sciences Building, Foresterhill, Aberdeen, UK

4 Talmedica Ltd, St Crispin House, St Crispin Way, Haslingden, Rossendale, UK

5 Child Health, Royal Aberdeen Children's Hospital, Aberdeen AB25 2ZG, UK concentrations were measured $8 \mathrm{~h}$ after dosing when reviewed at 1, 2 and 3 weeks and $6 \mathrm{~h}$ after dosing when reviewed at 5 weeks. Sputum cysteamine concentration was also quantified at the 5-week assessment.

Results Seven of the ten participants reported adverse reactions typical of cysteamine, two participants discontinued intervention. Following the first 450-mg dose, mean (SD) maximum concentration $\left(C_{\max }\right)$ was $2.86(1.96) \mathrm{mg} / \mathrm{l}$, the time corresponding to $C_{\max }\left(T_{\max }\right)$ was $1.2(0.7) \mathrm{h}$, the half-life $\left(t_{1 / 2}\right)$ was $3.7(1.7) \mathrm{h}$, clearance (CL/F) 89.9 (30.5) $\mathrm{L} / \mathrm{h}$ and volume of distribution $\left(V_{\mathrm{d}} / F\right) 427$ (129) L. Cysteamine appeared to accumulate in sputum with a median (interquartile range) sputum:plasma cysteamine concentration ratio of $4.2(0.98-8.84)$.

Conclusion Oral cysteamine is absorbed and enters the bronchial secretions in patients with CF. Although adverse reactions were common, the majority of patients continued with cysteamine. Further trials are required to establish the risk benefit ratio of cysteamine therapy in CF.

\section{Key Points}

Oral cysteamine is absorbed after taken by adult patients with cystic fibrosis and accumulates in the bronchial secretions.

The pharmacokinetics of cysteamine in people with cystic fibrosis are similar to those reported for people with cystinosis.

Although seven of the ten patients experienced side effects typical of cysteamine and led to the withdrawal of one of the participants, the majority of participants continued taking cysteamine. 


\section{Introduction}

Cystic fibrosis (CF) is an autosomal recessively inherited multi-organ disease that affects 70,000-100,000 people globally [1]. Most CF morbidity and mortality results from chronic suppurative lung disease [2]. Many of the bacteria infecting the lungs in $\mathrm{CF}$ are frequently resistant to antibiotics [3] and grow in biofilms, further reducing antibiotic sensitivity [4]. These issues have led to calls for new antibiotic strategies targeting the biofilm and to increase the effectiveness of currently available antibiotics $[5,6]$.

We have previously described the in vitro antimicrobial, anti-biofilm and mucoactive properties of the aminothiol cysteamine as a monotherapy and synergy with CF guideline recommended antibiotics [7-9]. Overall these characteristics suggest that cysteamine could be beneficial in managing $\mathrm{CF}$ and warrants further investigation. Although cysteamine has been used to treat nephropathic cystinosis for several decades [7] and its pharmacokinetic and clinical profiles are established for cystinosis [7, 1012], there are no such data for cysteamine in CF. In patients with cystinosis, absorption of oral cysteamine may be decreased when taken with food [13], there is a $40 \%$ firstpass effect, cysteamine circulates bound to plasma proteins, and is metabolised to taurine and excreted in the urine and bile; cysteamine is also excreted in the urine as inorganic sulphate and taurine [7, 14]. Given the pancreatic and intestinal manifestations of $\mathrm{CF}$ and the increased drug metabolism/clearance reported [15] in CF it is not possible to extrapolate the pharmacokinetics of cysteamine in cystinosis to CF. Critically it is not known in CF whether orally administered cysteamine enters infected airways.

We report here a single-arm, phase 1/2a open label study conducted to confirm in people with $\mathrm{CF}$ that oral cysteamine is absorbed, to characterise the pharmacokinetic profile of cysteamine, to ascertain whether cysteamine enters bronchial secretions and to explore tolerability.

\section{Method}

This was a single-centre, single-group, open-lable trial of administering oral cysteamine to patients with $\mathrm{CF}$. Figure 1 depicts the study design.

\subsection{Participants}

Patients attending the adult $\mathrm{CF}$ clinic at Aberdeen Royal Infirmary, Aberdeen, UK were invited to participate. The inclusion criteria were: CF lung disease, aged $\geq 18$ years, weight $>50 \mathrm{~kg}$, clinically stable for $>4$ weeks, and females of child-bearing potential using a reliable form of contraception. The exclusion criteria were: hypersensitivity to cysteamine, excipients or penicillamine, transplant recipient, pregnancy, planned pregnancy, and breast feeding. The trial was co-sponsored by the University of Aberdeen and NHS Grampian, approved by Scotland-A Research Ethics Committee (14/SS/056), the Medicines and Healthcare products Regulatory Agency (EudraCT 2014-000284-40, CTA 21583/0220/001-0001) and registered with http:// www.clinicaltrials.gov (NCT02212431). Participants provided written informed consent.

\subsection{Intervention}

Oral cysteamine bitartrate $\left(\right.$ Cystagon ${ }^{\circledR}$, Orphan Europe, Henley-upon-Thames, UK) was administered at the dose licensed for cystinosis, i.e. $450 \mathrm{mg}$ four times a day (qds). Participants were commenced on $450 \mathrm{mg}$ once a day, with the dose being increased at weekly intervals to $450 \mathrm{mg}$ twice daily (bd), $450 \mathrm{mg}$ three times a day (tds) and $450 \mathrm{mg}$, which was administered for 2 weeks (Fig. 1). As recommended, participants took cysteamine just after/with food [16].

\subsection{Follow-Up and Data Collection}

Participants were assessed at face-to-face visits at recruitment/baseline, and 1, 2, 3, 5 and 6 weeks (Fig. 1). The recruitment/baseline assessment was conducted in an inpatient facility, all other assessments were conducted in an out-patient facility. At each assessment, blood was obtained for routine clinical haematology and biochemistry. Adverse reactions (ARs) and serious adverse events (SAEs) were ascertained at the 1-, 2-, 3-, 5- and 6-week visits [17].

\subsubsection{Plasma and Sputum Samples}

At recruitment/assessment blood samples were taken prior to and $0.5,1,1.5,2,2.5,3,4,6,8,10$ and $24 \mathrm{~h}$ after the first 450-mg cysteamine dose that was administered by a CF nurse specialist. Blood (5 ml) was collected into chilled sodium heparin tubes, stored on ice until centrifuged at $2000 \mathrm{~g}, 4^{\circ} \mathrm{C}$ for $10 \mathrm{~min}$, and the plasma stored at $-70{ }^{\circ} \mathrm{C}$ within $1 \mathrm{~h}$ of collection. Further blood samples were taken $3 \mathrm{~h}$ after the last dose of cysteamine at the 1-, 2-, 3- and 5 -week visits. After a planned review of the pharmacokinetic metrics of the first four participants, the 24-h sample after the first dose was made optional because plasma cysteamine was below the lower level of quantification. In addition, the timing of the blood sampling was changed to $8 \mathrm{~h}$ after last dosing for the 1-, 2- and 3-week assessments and after $6 \mathrm{~h}$ for the 5-week assessment. These revisions 
Fig. 1 Study schedule. od once a day, $b d$ twice a day, $t d s$ three times a day, $q d s$ four times a day

\section{Recruitment/baseline visit}

Informed consent.

Inclusion/exclusion criteria, weight, blood samples for haematology, biochemistry.

Commenced on Cysteamine $450 \mathrm{mg}$ od

Blood cysteamine baseline, 30mins, 1, 1.5, 2, 2.5, 3, 4, 6, 8, 10, (and 24) hours

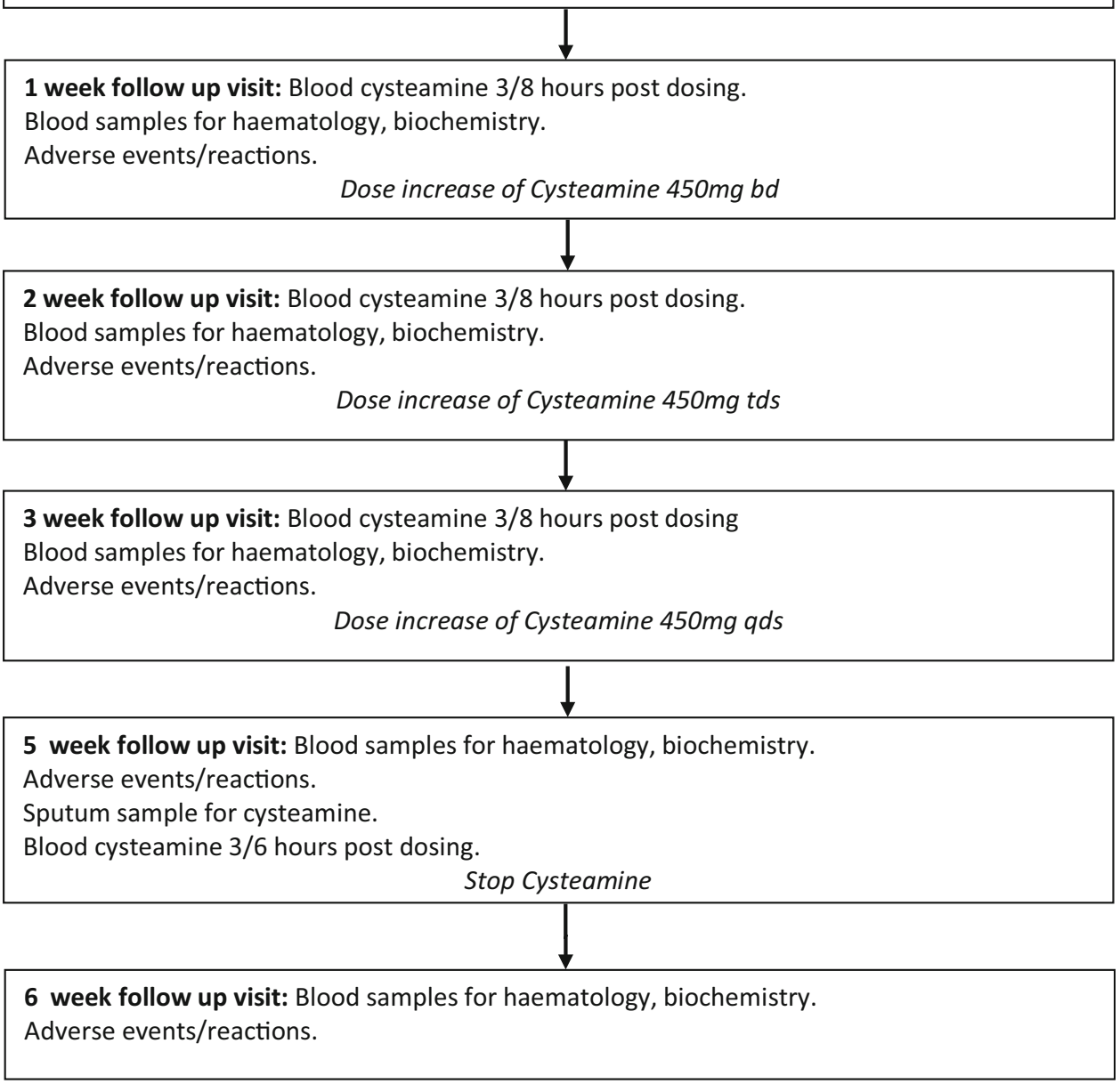

were agreed by the Trial Steering Committee. A spontaneously expectorated sample of sputum $(\sim 1 \mathrm{ml})$ was collected at the 5-week assessment immediately prior to blood sampling and stored at $-70{ }^{\circ} \mathrm{C}$ within an hour of collection.

\subsubsection{Quantification of Cysteamine}

Plasma and sputum cysteamine was quantified using protein precipitation and derivatisation followed by liquid chromatography with tandem mass spectrometric detection (LC-MS/MS) [18-20] by Covance Laboratories Ltd, Harrogate, UK. The validated method has a calibration range of $0.020-5.000 \mathrm{mg} / \mathrm{l}$. The analytical work was conducted in accordance with: UK Statutory Instrument 2004 No. 1031: The Medicines for Human Clinical Use (Clinical
Trials) Regulations 2004, Statutory Instrument 2004 No.994 and Guidelines on Good Clinical Practice: ICH E6: Good Clinical Practice CPMP/ICH/135/95 (July 1996).

\subsubsection{Statistical Considerations}

Primary outcomes were single-dose pharmacokinetic parameters. Secondary outcomes were sputum cysteamine concentration and tolerability. With 12 subjects the study had $80 \%$ power with $\alpha=0.05$ to detect a $\geq 1$ SD difference in the mean maximal plasma concentration $\left(C_{\max }\right)$ after first dose of cysteamine between cystinosis [10] and CF patients.

Pharmacokinetic parameters were identified using standard non-compartmental analysis from the single-dose cysteamine concentration against time profiles. $C_{\max }$ was 
the greatest measured level and $T_{\max }$ was the time corresponding to $C_{\max }$. The elimination rate constant $(k)$ was obtained by linear regression of the natural logarithm cysteamine concentration against time from the terminal portion of each profile (i.e. all concentrations after the peak concentration) and the half-life $\left(t_{1 / 2}\right)$ was the natural logarithm of two divided by the elimination rate constant. The area under the curve between time $t=0$ and the last sample (Cfin-the final concentration) was calculated using the trapezoidal rule $\left(\mathrm{AUC}_{0-\mathrm{Cfin}}\right)$. The area under the curve (AUC) was the $\mathrm{AUC}_{0-\mathrm{C} f i n}$ plus the residual area (the final measured concentration divided by the elimination rate constant). The residual area ranged from 4.1 to $13.4 \%$ of the AUC for all participants. Bioavailability $(F)$ of cysteamine tablets was not calculated, hence clearance (CL) and volume of distribution $\left(V_{\mathrm{d}}\right)$ are reported as the respective parameters divided by $F\left(\mathrm{CL} / F\right.$ and $V_{\mathrm{d}} / F$, respectively). $\mathrm{CL} / F$ was obtained by dividing the dose by the AUC and then dividing this value by the elimination rate constant provided $V_{\mathrm{d}} / F$. SPSS, v23.0. (Armonk, NY, USA) was used for data analyses.

\section{Results}

Of the 65 patients attending the CF clinic, 32 were eligible and invited to participate. Ten people participated between September 2014 and February 2015, 22 declined to participate. Two participants discontinued the intervention. Figure 2 outlines flow through the trial. The clinical characteristics of the participants are presented in Table 1.

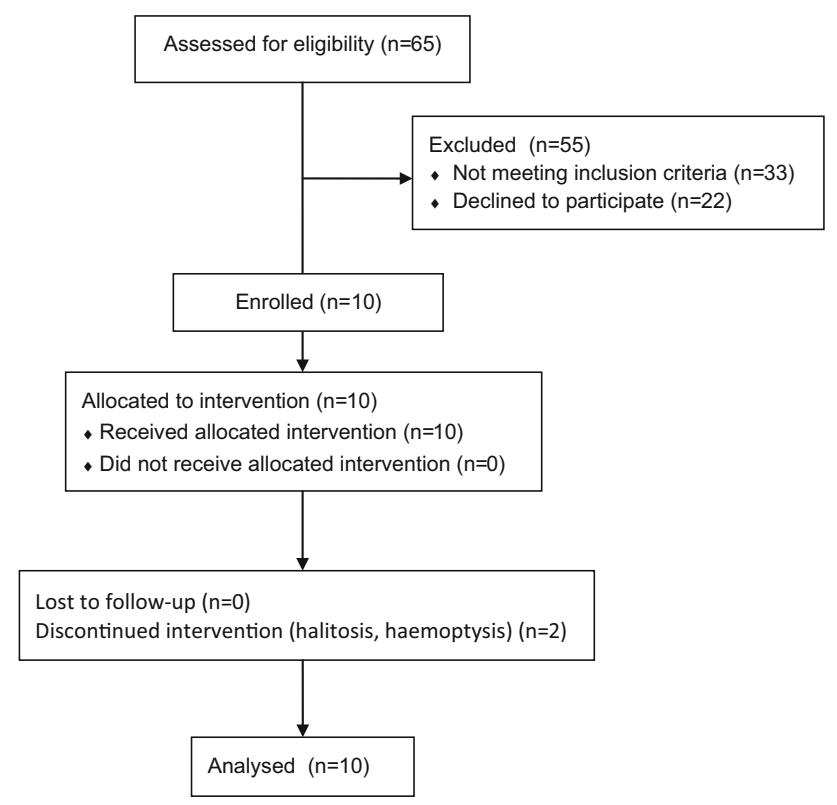

Fig. 2 CONSORT flow diagram

\subsection{Plasma Cysteamine}

Serial plasma cysteamine concentrations after the first 450-mg cysteamine dose were quantified in nine participants, venous access issues prevented measurement in one participant. A median (interquartile range, IQR) of 9 (7-10) time points were used to calculate half-life. Plasma cysteamine concentrations and derived pharmacokinetic parameters are presented in Fig. 3 and Table 2. Following 450-mg once a day $(n=8)$, twice a day $(n=9)$, three times a day $(n=8)$ and four times a day $(n=8)$ dosing the mean (SD) plasma cysteamine concentrations were 0.47 (0.26), 0.60 (0.34), 0.85 (0.73) and $0.47(0.34) \mathrm{mg} / \mathrm{l}$, respectively. Four of the eight patients with measured concentrations on four times a day dosing had lower concentrations than three times a day dosing although their pill counts suggested good adherence.

\subsection{Sputum Cysteamine}

Six participants were able to expectorate sputum samples immediately before blood sampling at the 5-week assessment. Cysteamine was detected in all of these six sputum samples, median (IQR) sputum cysteamine concentration was $0.84(0.41-2.10) \mathrm{mg} / \mathrm{l}$. The median (IQR) ratio of sputum to plasma cysteamine concentration was 4.2 (0.98-8.84).

\subsection{Tolerability}

Adherence as determined by pill counting appeared to be good, with median (IQR) adherence for the once, twice, three times and four times daily dosing being $100 \%$ (100-100), $100 \%$ (93-100), $98 \%(95-100)$ and $98 \%$ (79-100), respectively.

Two participants discontinued the intervention: one during the first week because of halitosis (an adverse reaction [AR] related to cysteamine); the second during week three because of recurrence of haemoptysis that continued after ceasing medication (an AR unrelated to cysteamine). There were two SAEs: one participant was hospitalised twice with infective exacerbations of CF lung disease. These SAEs were deemed unrelated to cysteamine. There were no deaths. Gastrointestinal ARs (anorexia, nausea, vomiting) were reported by four participants, for three this was either short lived (2 days), very mild or resolved with an increased dose of proton pump inhibitor; for one participant symptoms persisted throughout the trial. Neurological ARs (lethargy, somnolence, forgetfulness) were reported by three participants. One participant reported a transient rash after commencing cysteamine (a possible AR). Seven participants reported at least one AR. Two participants reported AEs judged unrelated to cysteamine. One participant reported no AE/ARs. 
Table 1 Baseline clinical characteristics of participating patients

Fig. 3 Presentation of data on mean (SD) plasma cysteamine concentrations in the first $24 \mathrm{~h}$ after an oral dose of $450 \mathrm{mg}$ $(n=9)$. Data points for $\mathrm{t} 0-10 \mathrm{~h}$ are mean (SD) of $n=9$ and for $t=24 \mathrm{~h}$, mean (SD) of $n=7$

Clinical characteristic Value

Age, years, median (interquartile range)

$21.5(19.8-31.8)$

Female, $n(\%)$

CF genotype: Phe 508del/Phe 508del, $n(\%)$

$6(60 \%)$

$8(80 \%)$

$2(20 \%)$

CF genotype: Phe 508del/-

$22.1(20.3-23.9)$

Body mass index, $\mathrm{kg} / \mathrm{m}^{2}$, mean (95\% confidence interval)

$32 \%(26-49)$

$$
\text { range) }
$$

Concomitant medication, $n(\%)$

Pancreatic enzyme replacement

Azithromycin

Inhaled antibiotic

$4(40 \%)$

Nebulised recombinant DNAse

$3(30 \%)$

Nebulised $4 \%$ saline

$1(10 \%)$

Ivacaftor

$1(10 \%)$

Sputum culture, $n(\%)$

Staphylococcus aureus

$3(30 \%)$

Pseudomonas aeruginosa

$6(60 \%)$

Stenotrophomonas maltophilia

\section{Plasma \\ Cysteamine \\ (mg/L)}

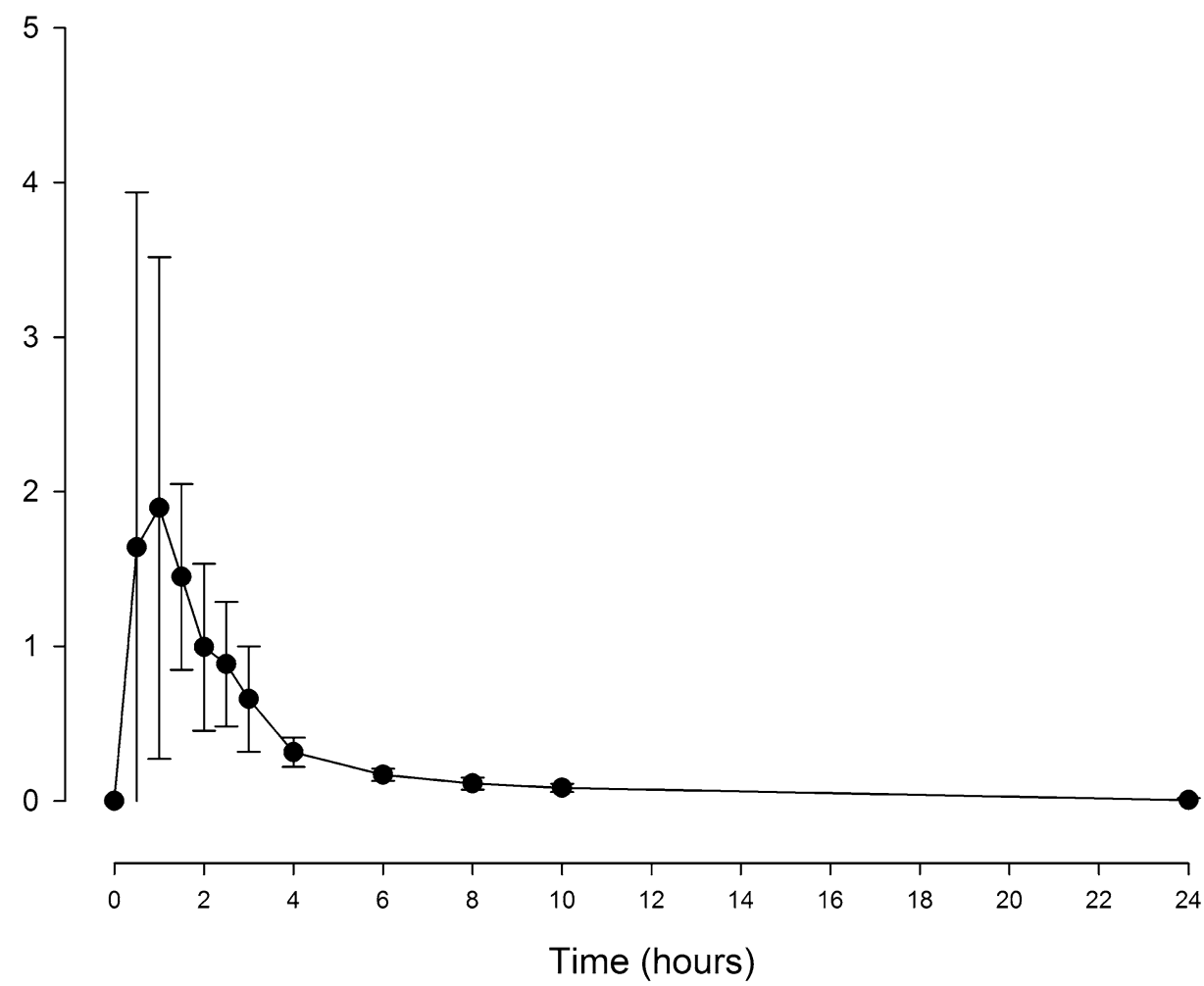


Table 2 Plasma pharmacokinetic parameters after a single dose of $450 \mathrm{mg}$ cysteamine

\begin{tabular}{|c|c|}
\hline Parameter & Value $^{a}$ \\
\hline$C_{\max }(\mathrm{mg} / \mathrm{l})$ & $2.86(1.95)$ \\
\hline$T_{\max }(\min )$ & $72(42)$ \\
\hline$t_{1 / 2}(\min )$ & $222(102)$ \\
\hline $\mathrm{AUC}_{0-\infty}(\mathrm{mg} \cdot \mathrm{min} / \mathrm{l})$ & $9.62(2.02)$ \\
\hline $\mathrm{CL} / \mathrm{F}(1 / \mathrm{min})$ & $1.50(0.51)$ \\
\hline$V_{\mathrm{d}} / F(1)$ & 427 (129) \\
\hline$V_{\mathrm{d}} / F\left(1 / \mathrm{kg}^{\mathrm{b}}\right)$ & $7.1(2.1)$ \\
\hline
\end{tabular}

$C_{\max }$ maximum concentration, $T_{\max }$ time corresponding to $C_{\max }, t_{1 / 2}$ half-life, $C L / F$ clearance, $V_{d} / F$ volume of distribution

${ }^{a}$ Values expressed as mean (SD)

b Actual body weight

\section{Discussion}

We have shown that in adults with CF, oral cysteamine is absorbed and enters the bronchial secretions with a higher concentration than plasma values. Although ARs were common, most participants continued the intervention.

Most of the plasma pharmacokinetic parameters reported here in patients with $\mathrm{CF}$ are similar to those reported in cystinosis [10-12]. In patients with cystinosis, Belldina reported a two-compartment model with $C_{\max } 2.7 \mathrm{mg} / \mathrm{l}$ and $T_{\max } 84 \mathrm{~min}$ [11], Langman reported $C_{\max } 2.73 \mathrm{mg} / \mathrm{l}, T_{\max }$ $72 \mathrm{~min}$ and clearance $1.33 \mathrm{l} / \mathrm{min}$ [10]. In our CF patients volume of distribution and $t_{1 / 2}$ were greater than those reported after similar single dosing in people with cysti$\operatorname{nosis}\left(V_{\mathrm{d}} / F=180 \mathrm{~L}, \mathrm{SD} 112, t_{1 / 2}=90 \mathrm{~min}, \mathrm{SD} 23\right)$ [10], suggesting that whilst cysteamine absorption is similar in cystinosis and $\mathrm{CF}$, distribution and elimination differ. The volume of distribution of cysteamine in people with $\mathrm{CF}$ exceeds extracellular fluid volume, being similar to that reported for aminoglycosides in CF. For aminoglycosides this has been attributed to the compromised nutritional status of patients with CF [15]. The between-subject variability of pharmacokinetic parameters as evidenced by greater standard deviation values we report in $\mathrm{CF}$ patients is greater than that reported for cystinosis [10-12]. This may be a consequence of cysteamine being taken with food, a factor known to cause wide variation in cysteamine $T_{\max }$ and $C_{\max }$ [13]. Given the importance of maintaining nutritional status in $\mathrm{CF}$, it was not considered appropriate for subjects to fast. Consideration of the physical properties of cysteamine (acid dissociation constant, $\mathrm{pKa}=10.4$, partition coefficient, $\log P=0.01$ ) [21] predicts that cysteamine concentration in the acidic bronchial secretions of CF (pH 6.0-6.9) [22] should be higher than in the systemic circulation, which is consistent with our findings. A study published whilst our study was being conducted reported that oral cysteamine was absorbed by CF patients [23]. Although similar in size to our study $(n=10)$, this study differed somewhat: participants were younger, had milder lung disease, were all Phe508del homozygous and plasma cysteamine was quantified at baseline, 2 and $6 \mathrm{~h}$ for nine subjects and hourly for $6 \mathrm{~h}$ for one subject.

The side effects of cysteamine are well documented for cystinosis and consistent with what we observed in our CF patients, with seven participants $(70 \%)$ reporting ARs. Gastrointestinal ARs were most common and probably similar incidence to those reported for cystinosis. More participants reported neurological ARs (lethargy, somnolence, forgetfulness) than would be expected from the cystinosis data. Although pill counts indicated good adherence, the lower plasma levels after qds dosing compared to tds dosing suggested greater adherence with tds dosing. Anecdotally, participants spontaneously volunteered that they found qds dosing very difficult to incorporate into their daily routine. It is possible that for the qds dosing some of the capsules taken from the bottles were not taken. In patients with cystinosis, adherence is also poor in up to $70-80 \%$ of patients because of qds dosing and side effects [24-26].

The strengths of the current study are that the pharmacokinetic characteristics of cysteamine were established by serial measurements of plasma cysteamine after single dosing. When compared with UK CF Registry data the patients participating in the current study had a greater predominance of Phe508del homozygotes, lower lung function, but similar BMI, Pseudomonas infection and treatment modalities [27]. Quantification of sputum cysteamine was a further strength. Although sufficient to characterise pharmacokinetic parameters the small number of participants is a weakness. Our aim was to recruit 12 patients; however, ten volunteered, but this was in line with pre-study expectations given the small clinic size and the intensive nature of the study.

\section{Conclusion}

In conclusion, we have shown that in people with $\mathrm{CF}$, oral cysteamine is absorbed and preferentially accumulates in sputum. However, randomised controlled trials are required to establish whether and under what circumstances cysteamine could be used in the management of CF.

Acknowledgments Trial Steering Committee: Dr Steve Cunningham (chair), Dr Stephen Bourke, Dr Gordon MacGregor.

We are grateful to all the participants who took part in the study. We are also grateful to the support from the Clinical Trials Pharmacy at Aberdeen Royal Infirmary.

Author contributions GD as Chief Clinical Investigator contributed to study inception, obtained funding, contributed to the 
recruitment of patients, collection of samples, data analysis and manuscript preparation.

SS and KG contributed to study design, patient recruitment, data collection and manuscript preparation.

DFP and ED conducted the microbiological aspects of the study, and contributed to data analysis and manuscript preparation.

HC contributed to all pharmacological aspects of the study, study design, pharmacokinetic data analysis and manuscript preparation.

SC and JN contributed all aspects of trial management, study design, conduct of trial, data analysis and manuscript preparation.

DO' $\mathrm{N}$ contributed to study inception, obtained funding, and contributed to data analysis and manuscript preparation.

\section{Compliance with Ethical Standards}

All subjects provided written informed consent.

Conflict of interest KG and SS received funding from Novabiotics to attend an educational meeting in June 2015.

$\mathrm{HC}$ received an honorarium from the University of Aberdeen for his work on the study.

DFP reports personal fees from NovaBiotics Ltd, outside the submitted work; DFP has a patent Treatment of Mycobacterium abscessus infections GB14832P pending.

ED reports grants from NovaBiotics Ltd, outside the submitted work. DO'N reports personal fees and other from NovaBiotics Ltd, from null, outside the submitted work; DO'N has a patent Biofilms PCT/ GB2010/000631 issued, a patent Cysteamine and antibiotics PCT/ GB2011/001721 issued, a patent Cysteamine for treating yeasts and moulds B1416727.4, US62/053,523 pending, and a patent Treatment of Mycobacterium abscessus infections GB14832P pending.

Role of the funding source This study was funded by Health Sciences Scotland (West Medical Building, University Avenue, Glasgow G12 8QQ. UK) and the Cystic Fibrosis Trust (One Aldgate, London. EC3N 1RE. UK). The funders did not contribute to study design, data collection, analysis, this report or the decision to publish.

Open Access This article is distributed under the terms of the Creative Commons Attribution-NonCommercial 4.0 International License (http://creativecommons.org/licenses/by-nc/4.0/), which permits any noncommercial use, distribution, and reproduction in any medium, provided you give appropriate credit to the original author(s) and the source, provide a link to the Creative Commons license, and indicate if changes were made.

\section{References}

1. World Health Organization. The molecular genetic epidemiology of cystic fibrosis. Report of a joint meeting of WHO/ECFTN/ $\mathrm{ICF}(\mathrm{M}) \mathrm{A} / \mathrm{ECFS}$. WHO/HGN/CF/WG/04.02. Geneva, WHO/ HGN/CF/WG, 2004.

2. Goss $\mathrm{CH}$, Quittner AL. Patient-reported outcomes in cystic fibrosis. Proc Am Thorac Soc. 2007;4:378-86.

3. Pitt TL, Sparrow M, Warner M, Stefanidou M. Survey of resistance of Pseudomonas aeruginosa from UK patients with cystic fibrosis to six commonly prescribed antimicrobial agents. Thorax. 2003;58:794-6.

4. Stewart PS, Costerton JW. Antibiotic resistance of bacteria in biofilms. Lancet. 2007;358:135-8.

5. Bals R, Hubert D, Tümmler B. Antibiotic treatment of CF lung disease: from bench to bedside. J Cystic Fibrosis. 2011;10(Suppl 2):S146-51.
6. Hoiby N. New antimicrobials in the management of cystic fibrosis. J Antimicrob Chem. 2002;49:235-8.

7. Besouw M, Masereeuw R, van den Heuvel L, Levtchenko E. Cysteamine: an old drug with new potential. Drug Discov Today. 2013;18(15/16):785-92.

8. Charrier C, Rodger C, Robertson J, Kowalczuk A, Shand N, Fraser-Pitt D, Mercer D, O’Neil D. Cysteamine (Lynovex ${ }^{\circledR}$ ), a novel mucoactive antimicrobial \& antibiofilm agent for the treatment of cystic fibrosis. Orphanet J Rare Dis. 2014;9:189.

9. Devereux G, Fraser-Pitt, Robertson J, Devlin E, Mercer D, O'Neil D. Cysteamine as a future intervention in cystic fibrosis against current and emerging pathogens: a patient-based ex vivo study confirming its antimicrobial and mucoactive potential in sputum. EBioMedicine. 2015. doi:10.1016/j.ebiom.2015.08.018.

10. Langman CB, Greenbaum LA, Sarwal M, Grimm P, Niaudet P, Deschenes G, Cornelissen E, Morin D, Cochat P, Matossian D, Gaillard S, Bagger MJ, Rioux P. A randomized controlled crossover trial with delayed-release cysteamine bitartrate in nephropathic cystinosis: effectiveness on white blood cell cystine levels and comparison of safety. Clin J Am Soc Nephrol. 2012;7:1112-20.

11. Belldina EB, Huang MY, Schneider JA, Brundage RC, Tracy TS. Steady-state pharmacokinetics and pharmacodynamics of cysteamine bitartrate in paediatric nephropathic cystinosis patients. J Clin Pharmacol. 2003;56:520-5.

12. Bouazza N, Tréluyer JM, Ottolenghi C, Urien S, Deschenes G, Ricquier D, Niaudet P, Chadefaux-Vekemans B. Population pharmacokinetics and pharmacodynamics of cysteamine in nephropathic cystinosis patients. Orphanet J Rare Dis. 2011;6:86.

13. Dohil R, Cabrera BL, Gangoiti J, Rioux P. The effect of food on cysteamine bitartrate absorption in healthy participants. Clin. Pharmacol. Drug Dev. 2012;1:170-4.

14. Salvador RA, Davison C, Smith PK. Metabolism of cysteamine. J Pharm Exp Therap. 1957;121:258-65.

15. Touw DJ, Vinks AA, Mouton JW, Horrevorts AM. Pharmacokinetic optimisation of antibacterial treatment in patients with cystic fibrosis. Current practice and suggestions for future directions. Clin Pharmacokinetics. 1998; 35:437-59.

16. Cystagon summary of product characteristics. http://www.ema. europa.eu/docs/en_GB/document_library/EPAR_-_Product_ Information/human/000125/WC500037764.pdf. Accessed 10 Apr 2016.

17. National Research Ethics Service. Safety and progress reports (CTIMPs). http://www.hra.nhs.uk/documents/2013/08/safetyreporting-ctimps-procedural-table.pdf. Accessed 10 Apr 2016.

18. Guan XHG, Dwivedi C, Matthees DP. A simultaneous liquid chromatography/mass spectrometric assay of glutathione, cysteine, homecysteine and their disulfides in biological samples. J Pharmaceut Biomed Anal. 2003;31:251-61.

19. Dohil R, Fidler M, Barshop B, Newbury R, Sellers Z, Deutsch R, Schneider J. Esomeprazole therapy for gastric acid hypersecretion in children with cystinosis. Pediatr Nephrol. 2005;20:1786-93.

20. Pavord ID, Pizzichini MM, Pizzichini E, Hargreave FE. The use of induced sputum to investigate airway inflammation. Thorax. 1997;52:498-501.

21. Drugbank. Cysteamine. http://www.drugbank.ca/drugs/DB00847. Accessed 10 Apr 2016.

22. Palmer KL, Aye LM, Whiteley M. Nutritional cues control Pseudomonas aeruginosa multicellular behavior in cystic fibrosis sputum. J Bacteriol. 2007;189:8079-87.

23. De Stefano D, Villella VR, Esposito S, Tosco A, Sepe A, De Gregorio F, Salvadori L, Grassia R, Leone CA, De Rosa G, Maiuri MC, Pettoello-Mantovani M, Guido S, Bossi A, Zolin A, Venerando A, Pinna LA, Mehta A, Bona G, Kroemer G, Maiuri L, Raia V. Restoration of CFTR function in patients with cystic 
fibrosis carrying the F508del-CFTR mutation. Autophagy. 2014;10:2053-74.

24. Levtchenko EN, van Dael CM, de Graaf-Hess AC, Wilmer MJ, van den Heuvel LP, Monnens LA, Blom HJ. Strict cysteamine dose regimen is required to prevent nocturnal cystine accumulation in cystinosis. Pediatr Nephrol. 2006;2006(21):110-3.

25. Besouw M, Blom H, Tangerman A, de Graaf-Hess A, Levtchenko E. The origin of halitosis in cystinotic patients due to cysteamine treatment. Mol Genet Metab. 2007;2007(91):228-33.
26. Besouw M, Levtchenko E. Growth retardation in children with cystinosis. Minerva Pediatr. 2010;62:307-14.

27. UK CF Registry, Annual Data Report 2014. http://www. cysticfibrosis.org.uk/media/1596846/RegistryReport2014.pdf. Accessed 102016. 